\title{
The jumping champions of the Farey series
}

\author{
by \\ Cristian Cobeli (Bucureşti), Kevin Ford (Urbana, IL) and \\ Alexandru Zaharescu (Bucureşti and Urbana, IL)
}

1. Introduction. Let $\mathcal{M}=\left\{\gamma_{1}, \ldots, \gamma_{M}\right\}$ be a set of real numbers ordered increasingly and let

$$
D(\mathcal{M})=\left\{\gamma_{i+1}-\gamma_{i}: 1 \leq i \leq M-1\right\}
$$

be the set of gaps between consecutive elements of $\mathcal{M}$. Usually we think of the elements of $D(\mathcal{M})$ as being arranged in ascending order, keeping in the list all the numbers with their multiplicities. A number $d$ is called a jumping champion of $\mathcal{M}$ (for short champion or JC) if the multiplicity of $d$ is largest among all elements of $D(\mathcal{M})$. According to [12], the term jumping champion was introduced by J. H. Conway in 1993.

Finding the JC of a set may be a very difficult problem. Certainly this is the case when $\mathcal{M}=\mathcal{P}_{n}$, the set of primes less than or equal to $n$. This problem has been investigated by Nelson [11], Erdös and Straus [2], and Harley [9]. Assuming conjectures for counts of prime $r$-tuples, Gallagher [4], [5] proved that $D\left(\mathcal{P}_{n}\right)$ approaches a Poisson distribution as $n \rightarrow \infty$. Odlyzko, Rubinstein and Wolf [12] give empirical and heuristic evidence that the JC for primes are 1,2, 4 and the primorials $6,30,210,2310, \ldots$ But as the technical difficulties encountered when dealing with consecutive primes are formidable enough, they cannot give a proof of this result even under the assumption of the prime $r$-tuple conjecture.

Our object here is to study the JC problem for the Farey series. Unlike the case of primes, in the case of Farey fractions we may prove, unconditionally, the asymptotics for the size of champions, and understand some of their arithmetical properties.

In the next two sections we introduce some terminology and state our main results, which are then proved in the following sections. At the end of the paper we attach samples from three tables showing the JC's for Farey sequences and related quantities.

2000 Mathematics Subject Classification: 11B57, 11N25.

Second author supported by National Science Foundation grant DMS-0196551. 
2. Notations. Let $\mathfrak{F}_{Q}$ be the sequence of Farey fractions of order $Q$. Taking into account the symmetry of $\mathfrak{F}_{Q}$ with respect to $1 / 2$, in what follows we will only work with $\mathcal{M}_{Q}=\mathfrak{F}_{Q} \cap[0,1 / 2]$. Then the number of gaps between consecutive elements of $\mathcal{M}_{Q}$ is $\left|D\left(\mathcal{M}_{Q}\right)\right|=\left(\left|\mathfrak{F}_{Q}\right|-1\right) / 2$.

Looking at $1 \leq Q \leq 9$, one finds that all the elements of $D\left(\mathcal{M}_{Q}\right)$ are distinct, so they all share the position of champion. When $Q=10$, we have

$$
\begin{aligned}
\mathcal{M}_{10} & =\left\{0, \frac{1}{10}, \frac{1}{9}, \frac{1}{8}, \frac{1}{7}, \frac{1}{6}, \frac{1}{5}, \frac{2}{9}, \frac{1}{4}, \frac{2}{7}, \frac{3}{10}, \frac{1}{3}, \frac{3}{8}, \frac{2}{5}, \frac{3}{7}, \frac{4}{9}, \frac{1}{2}\right\}, \\
D\left(\mathcal{M}_{10}\right) & =\left\{\frac{1}{10}, \frac{1}{90}, \frac{1}{72}, \frac{1}{56}, \frac{1}{42}, \frac{1}{30}, \frac{1}{45}, \frac{1}{36}, \frac{1}{28}, \frac{1}{70}, \frac{1}{30}, \frac{1}{24}, \frac{1}{40}, \frac{1}{35}, \frac{1}{63}, \frac{1}{18}\right\} .
\end{aligned}
$$

The gap $1 / 30$ appears twice in $D\left(\mathcal{M}_{10}\right)$, so it is the JC of $\mathfrak{F}_{10}$.

We know that if $a^{\prime} / q^{\prime}, a^{\prime \prime} / q^{\prime \prime}$ are consecutive fractions in $\mathfrak{F}_{Q}$, then the gap between them is

$$
\frac{a^{\prime \prime}}{q^{\prime \prime}}-\frac{a^{\prime}}{q^{\prime}}=\frac{1}{q^{\prime} q^{\prime \prime}},
$$

so we need to focus mainly on the pairs of consecutive denominators of Farey fractions. Moreover, there is a bijection (see [6]) between these pairs and the lattice points from

$$
\mathcal{T}_{Q}=\left\{\left(q_{1}, q_{2}\right): 1 \leq q_{1}, q_{2} \leq Q, q_{1}+q_{2}>Q, \operatorname{gcd}\left(q_{1}, q_{2}\right)=1\right\} .
$$

This motivates the following definitions. Denote by $h(D, Q)$ the number of gaps of length $1 / D$ in $\mathcal{M}_{Q}$; this can be written as

$$
\begin{aligned}
h(D, Q) & =\left|\left\{\left(q_{1}, q_{2}\right) \in \mathcal{T}_{Q}: q_{1} q_{2}=D, q_{1}<q_{2}\right\}\right| \\
& =|\{q \mid D: \operatorname{gcd}(q, D / q)=1, D / q<q \leq Q, D / q+q>Q\}| .
\end{aligned}
$$

It is plain that

$$
\left|D\left(\mathcal{M}_{Q}\right)\right|=\sum_{D \geq 1} h(D, Q) .
$$

Then any JC is a solution of the maximum problem

$$
M(Q)=\max _{D} h(D, Q) .
$$

We set

$$
\operatorname{Champs}(Q)=\{D: h(D, Q)=M(Q)\} .
$$

Usually we refer to the elements of $\operatorname{Champs}(Q)$ as being champions, although, strictly speaking, the champions are the inverses of the elements of Champs $(Q)$. Next, we denote by $H(Q)$ the number of distinct gaps (participants in the competition for JC), i.e.

$$
H(Q)=|\{D: h(D, Q) \geq 1\}| .
$$


Then we define $H_{r}(Q)$ to be the number of gaps with multiplicity $r$, that is,

$$
H_{r}(Q)=|\{D: h(D, Q)=r\}| .
$$

Clearly $H(Q)=\sum_{r \geq 1} H_{r}(Q)$. We also consider $G_{r}(Q)$, the number of gaps with multiplicity $\geq r$, or

$$
G_{r}(Q)=|\{D: h(D, Q) \geq r\}|=\sum_{i \geq r} H_{i}(Q) .
$$

Note that $G_{1}(Q)=H(Q)$. There is another equivalent definition of $h(D, Q)$ which is more convenient in our problems. Let

$$
\beta=\beta(D, Q)= \begin{cases}\sqrt{D / Q^{2}} & \text { if } D \geq \frac{1}{4} Q^{2}, \\ \frac{1}{2}\left(1+\sqrt{1-4 D / Q^{2}}\right) & \text { if } D<\frac{1}{4} Q^{2} .\end{cases}
$$

Then

$$
h(D, Q)=|\{q \mid D: \operatorname{gcd}(q, D / q)=1, \beta Q<q \leq Q\}| .
$$

In other words, $h(D, Q)$ counts certain divisors of $D$ lying in a short interval (note that $\beta \geq 1 / 2$ ). To study divisors of numbers in short intervals, Tenenbaum [14] introduced the functions

$$
\begin{aligned}
\tau(n, y, z) & =|\{d \mid n: y<d \leq z\}|, \\
H(x, y, z) & =|\{n \leq x: \tau(n, y, z) \geq 1\}| .
\end{aligned}
$$

We will be more concerned with slight variations of these, namely

$$
\begin{aligned}
\tau^{*}(n, y, z) & =|\{d \mid n: \operatorname{gcd}(d, n / d)=1, y<d \leq z\}|, \\
H^{*}(x, y, z) & =\left|\left\{n \leq x: \tau^{*}(n, y, z) \geq 1\right\}\right| .
\end{aligned}
$$

Tenenbaum [14] (see also Theorem 21 of [7]) has determined the approximate growth of $H(x, y, z)$. If $\alpha, \beta$ are fixed with $0<\alpha<\beta \leq 1$, we have

$$
H\left(x^{2}, \alpha x, \beta x\right) \ll \frac{x^{2}}{(\log x)^{\theta} \sqrt{\log \log x}},
$$

where $\theta=1-(1+\log \log 2) / \log 2=0.08607 \ldots$ A simple modification of the proof of Tenenbaum's lower bound yields the following. If $0<\alpha<\beta \leq 1$ and $0 \leq \gamma<\delta \leq 1$, then

$$
\begin{aligned}
H^{*}\left(\delta x^{2}, \alpha x, \beta x\right)-H^{*} & \left(\gamma x^{2}, \alpha x, \beta x\right) \\
& \gg \frac{x^{2}}{(\log x)^{\theta}} \exp \left(-c_{1} \sqrt{\log \log x \log \log \log x}\right)
\end{aligned}
$$

for $x \geq x_{0}(\alpha, \beta, \gamma, \delta)$ and for some positive constant $c_{1}$. Recently, the second author [3] has improved Tenenbaum's bounds for $H(x, y, z)$. These new results imply that the right sides of (2) and (3) may be replaced by

$$
\frac{x^{2}}{(\log x)^{\theta}(\log \log x)^{3 / 2}} \text {. }
$$


Throughout, variables $c_{1}, c_{2}, \ldots$ denote positive absolute constants. We also let $\omega(n)$ be the number of distinct prime factors of $n$ and let $[x]$ be the greatest integer $\leq x$.

3. Statements of results. We raise two problems related to the JC problem for the Farey series. The first is to estimate $M(Q)$ and determine the multiplicative structure of the numbers in $\operatorname{Champs}(Q)$. The second is to estimate the quantities $H(Q), H_{r}(Q)$ and $G_{r}(Q)$.

THEOREM 1. We have

$$
M(Q)=\exp \left(2 \log 2 \frac{\log Q}{\log \log Q}+O\left(\frac{\log Q}{(\log \log Q)^{2}}\right)\right) .
$$

Corollary 1. If $D \in \operatorname{Champs}(Q)$ then

$$
\omega(D)=2 \frac{\log Q}{\log \log Q}+O\left(\frac{\log Q}{(\log \log Q)^{2}}\right) .
$$

Since

$$
\omega(n) \leq 2 \frac{\log Q}{\log \log Q}+O\left(\frac{\log Q}{(\log \log Q)^{2}}\right) \quad \text { for all } n \leq Q^{2},
$$

the JC have close to the maximum possible number of prime factors for integers of their size. In particular, most of the prime factors of $D \in \operatorname{Champs}(Q)$ are small.

Corollary 2. If $R / \log Q \rightarrow \infty$ as $Q \rightarrow \infty$, then almost all the prime factors of $D$ are $\leq R$.

An interesting problem would be to bound the largest prime factor of any $D \in \operatorname{Champs}(Q)$.

For convenience in stating the next results, let

$$
L(x)=\exp \left(c_{1} \sqrt{\log \log x \log \log \log x}\right)
$$

be one of the factors in Tenenbaum's lower bound (3) for $H(x, y, z)$.

THEOREM 2. We have

$$
\frac{Q^{2}}{(\log Q)^{\theta} L(Q)} \ll H(Q) \ll \frac{Q^{2}}{(\log Q)^{\theta} \sqrt{\log \log Q}} .
$$

The new results of [3] imply that

$$
H(Q) \asymp \frac{Q^{2}}{(\log Q)^{\theta}(\log \log Q)^{3 / 2}},
$$

where as usual $\asymp$ means both $\ll$ and $\gg$. 
Theorem 3. For any $r \geq 2$, we have

$$
G_{r}(Q) \ll Q^{2}\left(\frac{e \log 2\left(\log \log Q+c_{2}\right)}{\log r}\right)^{\log r / \log 2} .
$$

Notice that the upper bound given by Theorem 3 is useless when $r \ll$ $(\log Q)^{\xi e \log 2}$, where $\xi \log \xi=\theta / e$, since by Theorem 2 it follows that

$$
G_{r}(Q) \leq G_{1}(Q) \ll \frac{Q^{2}}{(\log Q)^{\theta} \sqrt{\log \log Q}} .
$$

We give two lower bounds for $G_{r}(Q)$.

TheOREM 4. Let $Q$ be sufficiently large. Suppose $l$ is an integer with $1 \leq l \leq \log Q /\left(\log \log Q+c_{3}\right)$, and put

$$
r=\left[\frac{1}{l}\left(\begin{array}{c}
2 l \\
l
\end{array}\right)\right], \quad K=c_{4}(2 \log \log Q+2 l) \log (2 \log \log Q+2 l) .
$$

Then

$$
G_{r}(Q) \geq c_{5} \frac{Q^{2}}{(\log Q)^{\theta} L(Q)\left({\underset{2}{2 \log \log Q]+2 l+2}}_{2 l+2}\right) K^{2 l+2}} .
$$

For example, the case $l=1, r=2$ gives

$$
G_{2}(Q) \gg \frac{Q^{2}}{(\log Q)^{\theta} L(Q)} \cdot \frac{1}{(\log \log Q)^{8}(\log \log \log Q)^{4}} .
$$

The results of [3] allow us to replace $L(Q)$ in Theorem 4 by $(\log \log Q)^{3 / 2}$.

By Theorems 2 and 4 , if $l=o\left(\frac{\log \log Q}{\log \log \log Q}\right)$, equivalently $r=e^{o\left(\frac{\log \log Q}{\log \log \log Q}\right)}$, it follows that

$$
G_{r}(Q)=\frac{Q^{2}}{(\log Q)^{\theta+o(1)}} .
$$

On the other hand, when $l / \log \log Q \rightarrow \infty$, we get $G_{r}(Q) \geq Q^{2} / l^{(2+o(1)) l}$. But since $l \sim \log r /(2 \log 2)$, this yields

$$
G_{r}(Q) \geq Q^{2}(\log r)^{-(1+o(1)) \log r / \log 2} .
$$

This should be compared with the upper bound in Theorem 3 .

A better lower bound of $G_{r}(Q)$ for intermediate $l$,

$$
\log \log Q \ll l \leq \frac{1}{2} \frac{\log Q}{\log \log Q},
$$

is given below.

TheOrem 5. Suppose $2 \log \log Q \leq l \leq \frac{1}{2} \frac{\log Q}{\log \log Q}$ and put

$$
r=\frac{1}{11 \log Q}\left(\begin{array}{c}
2 l \\
l
\end{array}\right) \text {. }
$$


For positive absolute constants $c_{6}, c_{7}$, we have

$$
G_{r}(Q) \geq c_{6} \frac{Q^{2}}{l^{5 / 2} \log ^{2} Q}\left(\frac{e}{2 l}\left(\log \frac{\log Q}{l \log l}-c_{7} \frac{\log \log l}{\log l}\right)\right)^{2 l} .
$$

In this middle range for $l$, putting together Theorems 3 and 5 , we obtain the following.

Corollary 3. Suppose $Q \geq Q_{0}$,

$$
\frac{\log r}{\log \log Q \log \log \log Q} \rightarrow \infty \quad \text { and } \quad \log r=(\log Q)^{o(1)} .
$$

Then

$$
G_{r}(Q)=Q^{2}\left(\frac{(e \log 2+o(1)) \log \log Q}{\log r}\right)^{\log r / \log 2} .
$$

An interesting problem would be to determine, if it exists, the limit

$$
\lim _{Q \rightarrow \infty} \frac{H_{r}(Q)}{H(Q)}
$$

for each fixed $r$. The problem of comparing $H_{r}(x, y, z)$ to $H(x, y, z)$ (where $\left.H_{r}(x, y, z)=|\{n \leq x: \tau(n, y, z)=r\}|\right)$ was studied by Tenenbaum [15] and more recently by Ford [3], but the results are not strong enough to answer our question. In particular, Theorem 1 of [15] requires $z \leq x^{1 /(r+1)}$. The same question can be asked for the ratio $G_{r}(Q) / H(Q)$. The results of [3] imply that $G_{r}(Q) \gg_{r} H(Q)$, this being a consequence of the estimate

$$
H^{*}\left(\delta x^{2}, \alpha x, \beta x\right)-H^{*}\left(\gamma x^{2}, \alpha x, \beta x\right) \asymp \frac{x^{2}}{(\log x)^{\theta}(\log \log x)^{3 / 2}}
$$

valid for every fixed quadruple $\alpha, \beta, \gamma, \delta$. Suppose $r \geq 2$ and let $p_{1}<\ldots<$ $p_{r}<1.1 p_{1}$, where $p_{1}, \ldots, p_{r}$ are primes. If $\tau\left(n, 2 Q /\left(3 p_{1}\right), 3 Q /\left(4 p_{1}\right)\right) \geq 1$, then $\tau\left(p_{1} \ldots p_{r} n, 2 Q / 3, Q\right) \geq r$ and consequently by Lemma 1 below,

$$
\begin{aligned}
G_{r}(Q) & \geq H^{*}\left(\frac{4 Q^{2}}{9 p_{1} \ldots p_{r}}, \frac{2 Q}{3 p_{1}}, \frac{3 Q}{4 p_{1}}\right)-H^{*}\left(\frac{Q^{2}}{4 p_{1} \ldots p_{r}}, \frac{2 Q}{3 p_{1}}, \frac{3 Q}{4 p_{1}}\right) \\
& \gg_{r} \frac{Q^{2}}{(\log Q)^{\theta}(\log \log Q)^{3 / 2}} \gg_{r} H(Q) .
\end{aligned}
$$

4. Simple inequalities. By (1), we immediately get the upper bound

$$
h(D, Q) \leq \tau^{*}(D, Q / 2, Q) \leq \tau(D, Q / 2, Q) .
$$

A useful lower bound is

$$
h(D, Q) \geq \begin{cases}\tau^{*}(D, 2 Q / 3, Q) & \text { if } Q^{2} / 4<D \leq 4 Q^{2} / 9 \\ 0 & \text { otherwise. }\end{cases}
$$

A consequence of these bounds is 
Lemma 1 . We have

$$
\begin{aligned}
H(Q) & \leq H^{*}\left(Q^{2}, Q / 2, Q\right) \leq H\left(Q^{2}, Q / 2, Q\right), \\
H_{r}(Q) & \leq G_{r}(Q) \leq\left|\left\{D \leq Q^{2}: \tau(D, Q / 2, Q) \geq r\right\}\right|, \\
H(Q) & \geq H^{*}\left(4 Q^{2} / 9,2 Q / 3, Q\right)-H^{*}\left(Q^{2} / 4,2 Q / 3, Q\right), \\
G_{r}(Q) & \geq\left|\left\{Q^{2} / 4<D \leq 4 Q^{2} / 9: \tau^{*}(D, 2 Q / 3, Q) \geq r\right\}\right| .
\end{aligned}
$$

Theorem 2 now follows immediately from (2), (3), and Lemma 1.

Suppose that $\omega(D)=k$. Then $h(D, Q) \leq \tau^{*}(D, Q / 2, Q) \leq \tau^{*}(D, 0, D)=$ $2^{k}$. We can do a little bit better using Sperner's Theorem. Let $S=\left\{p^{a}\right.$ : $\left.p^{a} \| D\right\}$. There is a natural bijection between the subsets of $S$ and divisors $q$ of $D$ satisfying $\operatorname{gcd}(q, D / q)=1$. Also, the relation $\subseteq$ defines a partial order on the subsets of $S$. In any chain of distinct subsets $R_{1} \subseteq \ldots \subseteq R_{m}$, at most one of the associated divisors is counted by $\tau^{*}(D, Q / 2, Q)$. Sperner's Theorem (e.g. [13, p. 732, fact \# 7]) tells us that the subsets of $S$ can be partitioned into $\left(\begin{array}{c}k \\ \lfloor k / 2\rfloor\end{array}\right)$ chains. We thus have the following.

Lemma 2. Let $\omega(D)=k$. Then

$$
h(D, Q) \leq\left(\begin{array}{c}
k \\
\lfloor k / 2\rfloor
\end{array}\right) \ll \frac{2^{k}}{\sqrt{k}} .
$$

The inequality in Lemma 2 is nearly best possible, at least if $k$ is not too large as a function of $Q$. For example, let $k$ be even, let $p_{1}, \ldots, p_{k-1}$ be distinct primes in the interval $\left(\left(\frac{2 Q}{3}\right)^{2 / k},\left(\frac{4 Q}{5}\right)^{2 / k}\right]$, let $p_{k}$ be a prime in the interval $\left(\frac{9}{16}\left(\frac{2 Q}{3}\right)^{2 / k}, \frac{9}{16}\left(\frac{4 Q}{5}\right)^{2 / k}\right]$, and set $D=p_{1} \ldots p_{k}$. Then $Q^{2} / 4<$ $D \leq 9 Q^{2} / 25$. Let $q$ be any product of $k / 2$ primes $p_{i}, 1 \leq i \leq k-1$. Then $2 Q / 3<q<4 Q / 5$. By $(8), q$ is counted in $h(D, Q)$, therefore

$$
h(D, Q) \geq\left(\begin{array}{c}
k-1 \\
k / 2
\end{array}\right)=\frac{1}{2}\left(\begin{array}{c}
k \\
k / 2
\end{array}\right) \gg \frac{2^{k}}{\sqrt{k}} .
$$

5. The size of JC-Proof of Theorem 1. We turn to the problem of finding the size of $M(Q)$ as $Q \rightarrow \infty$. Let $q_{j}$ be the $j$ th prime and suppose $q_{1} \ldots q_{m} \leq Q^{2}<q_{1} q_{2} \ldots q_{m+1}$. Then if $D \leq Q^{2}$, it follows that $\omega(D) \leq m$, and by Lemma $2, M(Q) \leq\left(\begin{array}{c}m \\ {[m / 2]}\end{array}\right) \leq 2^{m}$. Now $\theta\left(q_{m}\right) \leq 2 \log Q<\theta\left(q_{m+1}\right)$, where $\theta$ is the Chebyshev function. By the Prime Number Theorem (with classical error term), we have

$$
q_{m}=2 \log Q+O\left((\log Q) e^{-c 8 \sqrt{\log \log Q}}\right),
$$

which implies

$$
m=\frac{2 \log Q}{\log \log Q}+O\left(\frac{\log Q}{(\log \log Q)^{2}}\right) .
$$

This gives the upper bound. 
Let $A=\exp \left(c_{9} \frac{\log Q}{\log \log Q}\right)$, in which $c_{9}$ is some large constant. Suppose $q_{1} \ldots q_{2 n} \leq Q^{2} / A^{2}<q_{1} \ldots q_{2 n+2}$. By the Prime Number Theorem,

$$
n=\frac{\log Q}{\log \log Q}+O\left(\frac{\log Q}{(\log \log Q)^{2}}\right) .
$$

If $t$ is the product of any $n$ primes $\leq q_{2 n}$, then

$$
t=\frac{Q}{A} \exp \left(O\left(\frac{\log Q}{\log \log Q}\right)\right) .
$$

Choose $c_{9}$ so that for any such $t$,

$$
Q / A^{3 / 2} \leq q_{1} \ldots q_{n} \leq t \leq q_{n+1} \ldots q_{2 n} \leq Q / A^{1 / 2} .
$$

By the box principle, for some $z \in\left[Q / A^{3 / 2}, Q / A^{1 / 2}\right]$, the interval $[z, 1.1 z]$ contains $\gg \frac{1}{\log A}\left(\begin{array}{c}2 n \\ n\end{array}\right)$ numbers $t$. Let $s_{1}$ be a prime in $\left(\frac{2 Q / 3}{z}, \frac{0.7 Q}{z}\right]$ and put $D_{0}=q_{1} \ldots q_{2 n} s_{1}$. Such an $s_{1}$ exists because $Q / z \geq A^{1 / 2} \rightarrow \infty$ as $Q \rightarrow \infty$. Also $s_{1} \geq q_{2 n}$, thus $D_{0}$ has $\gg \frac{1}{\log A}\left(\begin{array}{c}2 n \\ n\end{array}\right)$ divisors in $(2 Q / 3,0.77 Q]$. Since $D_{0} \leq \frac{Q^{2}}{A^{2}} \frac{0.7 Q}{z} \leq \frac{Q^{2}}{A^{1 / 2}}$, there is a prime $s_{2} \neq s_{1}$ lying in $\left(\frac{Q^{2} / 4}{D_{0}}, \frac{Q^{2} / 3}{D_{0}}\right]$. Put $D=s_{2} D_{0}$. Using (8), we conclude that

$$
\begin{aligned}
h(D, Q) & \gg \frac{\left(\begin{array}{c}
2 n \\
n
\end{array}\right)}{\log A} \gg \frac{2^{2 n} \log \log Q}{\sqrt{n} \log Q} \\
& =\exp \left((2 \log 2) \frac{\log Q}{\log \log Q}+O\left(\frac{\log Q}{(\log \log Q)^{2}}\right)\right) .
\end{aligned}
$$

This estimate concludes the proof of Theorem 1 .

In order to prove Corollary 1 , we see that if $D$ is a champion, then $h(D, Q)=M(Q) \leq 2^{\omega(D)}$ and therefore $\omega(D) \geq(\log M(Q)) / \log 2$. This gives the lower bound. The upper bound comes from the proof of Theorem 1 .

6. The sizes of the prime factors of champions. Corollary 2 follows from the next lemma.

Lemma 3. Suppose $Q$ is large, $R>3 \log Q$ and $D \in \operatorname{Champs}(Q)$. Then the number of prime factors of $D$ that are $\geq R$ is

$$
\ll \frac{\log Q}{\log \log Q} \frac{1}{\log \left(\frac{R}{3 \log Q}\right)} .
$$

Proof. Let $\omega=\omega(D)$ and let $N$ be the number of prime factors of $D$ which are $\geq R$. Then by Corollary 1 and the Prime Number Theorem,

$$
q_{1} \ldots q_{\omega} \geq Q^{2} \exp \left(-c_{10} \frac{\log Q}{\log \log Q}\right)
$$


and $q_{\omega} \leq 3 \log Q$ if $Q$ is large. Since

$$
\begin{aligned}
Q^{2} & \geq D \geq q_{1} \ldots q_{\omega-N} R^{N} \geq q_{1} \ldots q_{\omega}\left(\frac{R}{q_{\omega}}\right)^{N} \\
& \geq Q^{2} \exp \left(-c_{10} \frac{\log Q}{\log \log Q}\right)\left(\frac{R}{3 \log Q}\right)^{N},
\end{aligned}
$$

we get the lemma.

Another corollary of Lemma 3 is that the champions are not too small.

Corollary 4. If $D \in \operatorname{Champs}(Q)$, then $D \gg Q^{2} / \log ^{3} Q$.

Proof. Assume $D \leq c_{11} Q / \log ^{3} Q$, where $c_{11}$ is a sufficiently small positive constant. If $c_{12}$ is large enough, then at most $\omega(D) / 10$ prime factors of $D$ are $\geq c_{12} \log Q$. Thus $D$ has a divisor $d \in\left(Q /\left(2 c_{12}^{2} \log ^{2} Q\right), Q /\left(2 c_{12} \log Q\right)\right]$. Since $Q / d>2 c_{12} \log Q$, there exists a prime number $s \in\left(\frac{2}{3} \frac{Q}{d}, \frac{Q}{d}\right]$ which does not divide $D$. Since $D s \leq Q^{2} / \log Q$, there exists a prime number $t \in$ $\left(0.24 \frac{Q^{2}}{D s}, 0.25 \frac{Q^{2}}{D s}\right]$ which does not divide $D$. Let $D^{\prime}=D s t \in\left(0.24 Q^{2}, 0.25 Q^{2}\right]$. By (1) and the inequalities $\beta\left(D^{\prime}, Q\right)<0.6<\beta(D, Q)$ and $2 Q / 3<s d \leq Q$, we have $h\left(D^{\prime}, Q\right) \geq M(Q)+1$. We have obtained a contradiction, which completes the proof of the corollary.

An interesting question arises. What is the approximate size range of $\tau^{*}(D, \beta Q, Q) / \tau^{*}(D, 0, D)$ for champions $D$ ? Is this ratio always $\gg(\log Q)^{-B}$ for some $B>0$ ?

7. Upper bounds on $G_{r}(Q)$. In this section we prove Theorem 3 . Let $l$ be the smallest integer with $\left(\begin{array}{c}l \\ {[l / 2]}\end{array}\right) \geq r$. Denote by $\pi_{k}(x)$ the number of integers $\leq x$ with exactly $k$ distinct prime factors. In 1917, Hardy and Ramanujan [8] proved the uniform upper bound

$$
\pi_{k}(x) \ll \frac{x\left(\log \log x+c_{2}\right)^{k-1}}{(k-1) ! \log x}
$$

for a constant $c_{2}$. This bound suffices for our purposes, even though more precise bounds for $\pi_{k}(x)$ are known (see e.g. the papers by Balazard [1] and Hwang [10] for a survey of results and further references).

By Lemmas 1 and 2,

$$
G_{r}(Q) \leq\left|\left\{D \leq Q^{2}: \omega(D) \geq l\right\}\right| \ll \sum_{k \geq l} \frac{Q^{2}}{\log Q} \cdot \frac{\left(\log \log Q+c_{2}\right)^{k-1}}{(k-1) !} .
$$

If $l-1 \leq \log \log Q+c_{2}$, the bound in Theorem 3 is trivial, because the right hand side of (3) is $\geq Q^{2}$ in that case. Otherwise, we write $z=\log \log Q+c_{2}$, 
$l-1=\beta z$, and $\beta \geq 1$. Then

$$
\begin{aligned}
\frac{\log Q}{Q^{2}} G_{r}(Q) & \ll \sum_{h \geq \beta z} \frac{z^{h}}{h !} \leq \frac{1}{\beta^{\beta z}} \sum_{h \geq \beta z} \frac{(\beta z)^{h}}{h !} \leq\left(\frac{e}{\beta}\right)^{\beta z} \\
& =\left(\frac{e\left(\log \log Q+c_{2}\right)}{l-1}\right)^{l-1} .
\end{aligned}
$$

Lastly, $l-1 \leq \log r / \log 2$ and the theorem follows.

\section{Lower bounds for $G_{r}(Q)$}

Proof of Theorem 4. First, the upper bound on $l$ gives $K \leq c_{13} \log Q$ and thus if $B=Q / K^{l+1}$, then

$$
\begin{aligned}
B & \geq Q \exp \left(-\left[1+\frac{\log Q}{\log \log Q+c_{3}}\right]\left(\log \log Q+\log c_{13}\right)\right) \\
& \geq \exp \left(c_{14} \frac{\log Q}{\log \log Q}\right)
\end{aligned}
$$

if $c_{3}$ is large enough. By (3), it follows that

$$
H^{*}\left(B^{2}, B / 2, B\right) \geq c_{15} \frac{B^{2}}{(\log B)^{\theta} L(B)} .
$$

Let

$$
\widetilde{H}=\left\{m \leq B^{2}: \tau^{*}(m, B / 2, B) \geq 1, \omega(m) \leq 2 \log \log B\right\} .
$$

By (9) and Stirling's formula,

$$
\begin{aligned}
|\widetilde{H}| & \geq H^{*}\left(B^{2}, B / 2, B\right)-\sum_{k>2 \log \log B} \pi_{k}\left(B^{2}\right) \\
& \geq c_{15} \frac{B^{2}}{(\log B)^{\theta} L(B)}-c_{16} \frac{B^{2}}{(\log B)^{2 \log 2-1}} \\
& \geq c_{17} \frac{B^{2}}{(\log B)^{\theta} L(B)} \geq c_{17} \frac{B^{2}}{(\log B)^{\theta} L(Q)} .
\end{aligned}
$$

Let $m \in \widetilde{H}$, and let $d \mid m, B / 2<d \leq B, \operatorname{gcd}(d, m / d)=1$. Also let

$$
\mathcal{J}=\left(\frac{1}{c_{18}}\left(\frac{Q^{2}}{B^{2}}\right)^{1 /(2 l+2)}, \frac{1.1}{c_{18}}\left(\frac{Q^{2}}{B^{2}}\right)^{1 /(2 l+2)}\right]=\left(\frac{K}{c_{18}}, \frac{1.1 K}{c_{18}}\right) .
$$

By hypothesis, $K / c_{18} \geq \frac{c_{4}}{c_{18}}(2 \log \log Q+2 l) \log (2 \log \log Q+2 l)$. By the Prime Number Theorem, if $c_{4} / c_{18}$ is sufficiently large, then $\mathcal{J}$ contains $\geq$ $2 \log \log Q+2 l>2 \log \log B+2 l$ primes. Thus $\mathcal{J}$ contains primes $s_{1}, \ldots, s_{2 l}$ that do not divide $m$. Then

$$
D_{0}:=m s_{1} \ldots s_{2 l} \leq\left(\frac{1.1}{c_{18}}\right)^{2 l} Q^{2} K^{-2} .
$$


Each product of $l$ of the primes $s_{i}$ lies in the interval

$$
\mathcal{I}=\left(\left(\frac{1}{c_{18}}\right)^{l} K^{l},\left(\frac{1.1}{c_{18}}\right)^{l} K^{l}\right] .
$$

By the box principle, for some $z \in \mathcal{I}$, the interval $(z, 1.1 z]$ contains at least $\left[\frac{1}{l}\left(\begin{array}{c}2 l \\ l\end{array}\right)\right]=r$ such products. Let $t$ be a prime in $\left(\frac{2 Q / 3}{d z}, \frac{Q}{1.1 d z}\right]$ that does not divide $D_{0}$. Since

$$
\frac{Q}{d z} \geq \frac{Q}{B z} \geq \frac{K^{l+1}}{\left(1.1 K / c_{18}\right)^{l}}=\left(\frac{c_{18}}{1.1}\right)^{l} K \geq K \quad \text { if } c_{18} \geq 1.1
$$

the interval contains $\geq 2 \log \log B+2 l+1$ primes, so $t$ exists. Then $t D_{0}$ has at least $r$ divisors $q$ with $\operatorname{gcd}\left(q, t D_{0} / q\right)=1$ and $2 Q / 3<q \leq Q$. Let $u$ be a prime in $\left(\frac{Q^{2} / 4}{t D_{0}}, \frac{4 Q^{2} / 9}{t D_{0}}\right]$ that does not divide $t D_{0}$. Since

$$
\frac{Q^{2}}{t D_{0}} \geq\left(\frac{c_{18}}{1.1}\right)^{2 l} K^{2} \frac{1.1 d z}{Q} \geq 0.55\left(\frac{c_{18}}{1.21}\right)^{l} K^{2} \frac{B K^{l}}{Q}>K
$$

if $c_{18} \geq 3, u$ exists. Finally let $D=u t D_{0} \in\left(Q^{2} / 4,4 Q^{2} / 9\right]$. By $(8), h(D, Q)$ $\geq \frac{1}{l}\left(\begin{array}{c}2 l \\ l\end{array}\right)$.

Since $D=u t s_{1} \ldots s_{2 l} m$, at most $\left(\begin{array}{c}\omega(D) \\ 2 l+2\end{array}\right)$ values of $m$ produce the same value of $D$. Since $\omega(D) \leq 2 l+2+2 \log \log Q$, (8) implies the result.

Proof of Theorem 5. Let $c_{19}$ be large enough so that $\pi\left(c_{19} l \log l\right) \geq 20 l$ for large $l$. Let $B=\left(\frac{Q}{c_{19} l \log l}\right)^{1 / l}$. By hypothesis, $B>c_{19} l \log l$. Let $s_{1}<$ $\ldots<s_{2 l}$ be any primes $\leq B$, and put $m=s_{1} s_{2} \ldots s_{2 l}$. There are $\left(\begin{array}{c}2 l \\ l\end{array}\right)$ products $s_{i_{1}} \ldots s_{i_{l}}$, each lying in $\left[1, B^{l}\right]$. Thus, for some $z \in\left[1, B^{l}\right]$, the interval $(z, 1.1 z]$ contains at least $r$ such products. Let $q$ be a prime in $\left(\frac{2 Q}{3 z}, \frac{Q}{1.1 z}\right]$ that does not divide $m$. Such a $q$ exists because $Q / z \geq Q / B^{l}=$ $c_{19} l \log l$ (i.e. $\pi\left(\frac{Q}{1.1 z}\right)-\pi\left(\frac{2 Q}{3 z}\right) \geq 2 l+2$ ). Let $s$ be a prime in $\left(\frac{Q^{2}}{4 q m}, \frac{4 Q^{2}}{9 q m}\right]$ that does not divide $q m$. Such an $s$ exists because $1.1 z \geq s_{1} \ldots s_{l} \geq m / B^{l}$ and thus

$$
\frac{Q^{2}}{q m} \geq \frac{Q z}{1.1 m} \geq \frac{1}{1.21} \frac{Q}{B^{l}} \geq \frac{c_{19}}{1.21} l \log l
$$

Put $D=s q m$, so $D \in\left(Q^{2} / 4,4 Q^{2} / 9\right]$. Also,

$$
\tau^{*}(D, 2 Q / 3, Q) \geq \tau^{*}(q m, 2 Q / 3, Q) \geq r,
$$

so $h(D, Q) \geq r$. Now each $D$ comes from at most $\left(\begin{array}{c}2 l+2 \\ 2\end{array}\right)$ values of $m$. Therefore, 


$$
\begin{aligned}
G_{r}(Q) & \geq \frac{1}{\left(\begin{array}{c}
2 l+2 \\
2
\end{array}\right)} \sum_{m} \sum_{\substack{2 Q /(3 z)<q \leq Q /(1.1 z) \\
\operatorname{gcd}(q, m)=1}} \sum_{Q^{2} /(4 q m)<s \leq 4 Q^{2} /(9 q m)} 1 \\
& \gg \frac{1}{l^{2}} \sum_{m} \sum_{q} \frac{Q^{2} /(q m)}{\log Q} \gg \frac{Q^{2}}{l^{2} \log ^{2} Q} \sum_{m} \frac{1}{m} .
\end{aligned}
$$

Lastly,

$$
\begin{aligned}
\sum_{m} \frac{1}{m} & \geq \frac{1}{(2 l) !} \sum_{s_{1} \leq B} \frac{1}{s_{1}} \sum_{\substack{s_{2} \leq B \\
s_{2} \neq s_{1}}} \frac{1}{s_{2}} \cdots \sum_{\substack{s_{2 l} \leq B \\
s_{2 l} \notin\left\{s_{1}, \ldots, s_{2 l-1}\right\}}} \frac{1}{s_{2 l}} \\
& \geq \frac{1}{(2 l) !} \sum_{s_{1} \leq B} \frac{1}{s_{1}} \sum_{\substack{q_{2} \leq s_{2} \leq B \\
s_{2}} \sum_{q_{2 l} \leq s_{2 l} \leq B} \frac{1}{s_{2 l}}} \frac{1}{(2 l) !}\left(\sum_{\substack{q_{2 l} \leq s \leq B \\
s}} \frac{1}{s}\right)^{2 l} \sum^{2 l} \frac{1}{\sqrt{l}}\left(\log \log B-\log \log q_{2 l}+O\left(\frac{1}{\log q_{2 l}}\right)\right)^{2 l}
\end{aligned}
$$

Now

$$
\log \log B=\log \frac{\log Q}{l}+O\left(\frac{\log l}{\log Q}\right)
$$

$\log \log q_{2 l}=\log \log (2 l(\log l+O(\log \log l)))=\log \log l+O\left(\frac{\log \log l}{\log l}\right)$

\begin{tabular}{|c|c|c|c|c|c|c|c|c|}
\hline$Q$ & $\left|D\left(\mathcal{M}_{Q}\right)\right|$ & $H(Q)$ & $H_{1}(Q)$ & $H_{2}(Q)$ & $H_{3}(Q)$ & $H_{4}(Q)$ & $H_{5}(Q)$ & $1 / \mathrm{JC}$ \\
\hline 2 & 1 & 1 & 1 & 0 & 0 & 0 & 0 & 2 \\
\hline 3 & 2 & 2 & 2 & 0 & 0 & 0 & 0 & 3,6 \\
\hline 4 & 3 & 3 & 3 & 0 & 0 & 0 & 0 & $4,6,12$ \\
\hline 5 & 5 & 5 & 5 & 0 & 0 & 0 & 0 & $5,10,12,15,20$ \\
\hline 6 & 6 & 6 & 6 & 0 & 0 & 0 & 0 & $6,10,12,15,20,30$ \\
\hline 7 & 9 & 9 & 9 & 0 & 0 & 0 & 0 & $\begin{array}{l}7,14,15,20,21,28, \\
30,35,42\end{array}$ \\
\hline 8 & 11 & 11 & 11 & 0 & 0 & 0 & 0 & $\begin{array}{l}8,14,20,21,24,28, \\
30,35,40,42,56\end{array}$ \\
\hline
\end{tabular}

and the theorem follows.

We conclude by proving Corollary 3. The upper bound comes from Theorem 3, while the lower bound follows from Theorem 5 , by taking $l=$ $\log r /(2 \log 2)+O(\log \log Q)$.

\section{Tables of champions}

Table 1. The $H$-values and the champions for a given $Q$ 
Table 1 (cont.)

\begin{tabular}{ccccccccl}
\hline$Q$ & $\left|D\left(\mathcal{M}_{Q}\right)\right|$ & $H(Q)$ & $H_{1}(Q)$ & $H_{2}(Q)$ & $H_{3}(Q)$ & $H_{4}(Q)$ & $H_{5}(Q)$ & $1 / \mathrm{JC}$ \\
\hline 180 & 4940 & 4481 & 4069 & 372 & 33 & 7 & 0 & $\begin{array}{l}8190,8580,9240, \\
9570,9660,10010, \\
10710\end{array}$ \\
\hline 181 & 5030 & 4594 & 4199 & 360 & 29 & 6 & 0 & $\begin{array}{l}8580,9240,9570, \\
9660,10010,10710\end{array}$ \\
\hline 182 & 5066 & 4608 & 4200 & 366 & 35 & 6 & 1 & 10010 \\
\hline 183 & 5126 & 4654 & 4235 & 374 & 38 & 6 & 1 & 10010 \\
\hline 184 & 5170 & 4686 & 4257 & 382 & 40 & 6 & 1 & 10010 \\
\hline 185 & 5242 & 4744 & 4301 & 396 & 40 & 6 & 1 & 10010 \\
\hline 186 & 5272 & 4754 & 4299 & 401 & 46 & 7 & 1 & 10010 \\
\hline 187 & 5352 & 4825 & 4365 & 402 & 50 & 7 & 1 & 10010 \\
\hline 188 & 5398 & 4860 & 4390 & 411 & 51 & 7 & 1 & 10010 \\
\hline 189 & 5452 & 4905 & 4426 & 420 & 51 & 7 & 1 & 10010 \\
\hline 190 & 5488 & 4918 & 4422 & 433 & 53 & 9 & 1 & 10010 \\
\hline
\end{tabular}

Table 2. Selected lists of champions

\begin{tabular}{cccl}
\hline Champion & Decomposition & No. of appearances & The values of $Q$ \\
\hline 102 & $2 \cdot 3 \cdot 17$ & 1 & 17 \\
\hline 104 & $2^{3} \cdot 13$ & 2 & 13,17 \\
\hline 105 & $3 \cdot 5 \cdot 7$ & 2 & 17,21 \\
\hline 110 & $2 \cdot 5 \cdot 11$ & 4 & $11-13,17$ \\
\hline 112 & $2^{4} \cdot 7$ & 1 & 17 \\
\hline 117 & $3^{2} \cdot 13$ & 2 & 13,17 \\
\hline 119 & $7 \cdot 17$ & 1 & 17 \\
\hline 120 & $2^{3} \cdot 3 \cdot 5$ & 1 & 17 \\
\hline 126 & $2 \cdot 3^{2} \cdot 7$ & 6 & $17-22$ \\
\hline 130 & $2 \cdot 5 \cdot 13$ & 2 & 13,17 \\
\hline 132 & $2^{2} \cdot 3 \cdot 11$ & 3 & $12,13,17$ \\
\hline 136 & $2^{3} \cdot 17$ & 1 & 17 \\
\hline 143 & $11 \cdot 13$ & 2 & 13,17 \\
\hline 144 & $2^{4} \cdot 3^{2}$ & 1 & 17 \\
\hline 153 & $3^{2} \cdot 17$ & 1 & 17 \\
\hline 154 & $2 \cdot 7 \cdot 11$ & 4 & $17,22-24$ \\
\hline 156 & $2^{2} \cdot 3 \cdot 13$ & 2 & 13,17 \\
\hline 165 & $3 \cdot 5 \cdot 11$ & 1 & 17 \\
\hline 168 & $2^{3} \cdot 3 \cdot 7$ & 5 & $24-28$ \\
\hline 170 & $2 \cdot 5 \cdot 17$ & 1 & 17 \\
\hline & & &
\end{tabular}


Table 2 (cont.)

\begin{tabular}{|c|c|c|c|}
\hline Champion & Decomposition & No. of appearances & The values of $Q$ \\
\hline 176 & $2^{4} \cdot 11$ & 1 & 17 \\
\hline 182 & $2 \cdot 7 \cdot 13$ & 2 & 17,26 \\
\hline 187 & $11 \cdot 17$ & 1 & 17 \\
\hline 195 & $3 \cdot 5 \cdot 13$ & 1 & 17 \\
\hline 198 & $2 \cdot 3^{2} \cdot 11$ & 7 & $22-28$ \\
\hline 1540 & $2^{2} \cdot 5 \cdot 7 \cdot 11$ & 5 & $55,59-62$ \\
\hline 1550 & $2 \cdot 5^{2} \cdot 31$ & 1 & 62 \\
\hline 1566 & $2 \cdot 3^{3} \cdot 29$ & 4 & $59-62$ \\
\hline 1650 & $2 \cdot 3 \cdot 5^{2} \cdot 11$ & 2 & 75,76 \\
\hline 1674 & $2 \cdot 3^{3} \cdot 31$ & 1 & 62 \\
\hline 1716 & $2^{2} \cdot 3 \cdot 11 \cdot 13$ & 5 & $55,59-62$ \\
\hline 1798 & $2 \cdot 29 \cdot 31$ & 1 & 62 \\
\hline 1938 & $2 \cdot 3 \cdot 17 \cdot 19$ & 4 & $59-62$ \\
\hline 1980 & $2^{2} \cdot 3^{2} \cdot 5 \cdot 11$ & 5 & $55,59-62$ \\
\hline 2310 & $2 \cdot 3 \cdot 5 \cdot 7 \cdot 11$ & 27 & $70-96$ \\
\hline 2520 & $2^{3} \cdot 3^{2} \cdot 5 \cdot 7$ & 5 & $72-76$ \\
\hline 2730 & $2 \cdot 3 \cdot 5 \cdot 7 \cdot 13$ & 18 & $91-108$ \\
\hline 3570 & $2 \cdot 3 \cdot 5 \cdot 7 \cdot 17$ & 20 & $107-126$ \\
\hline 3990 & $2 \cdot 3 \cdot 5 \cdot 7 \cdot 19$ & 15 & $114-118,121-126,133-136$ \\
\hline 4290 & $2 \cdot 3 \cdot 5 \cdot 11 \cdot 13$ & 1 & 130 \\
\hline 4620 & $2^{2} \cdot 3 \cdot 5 \cdot 7 \cdot 11$ & 5 & $132-136$ \\
\hline 4830 & $2 \cdot 3 \cdot 5 \cdot 7 \cdot 23$ & 1 & 138 \\
\hline 5460 & $2^{2} \cdot 3 \cdot 5 \cdot 7 \cdot 13$ & 9 & $140-148$ \\
\hline 6930 & $2 \cdot 3^{2} \cdot 5 \cdot 7 \cdot 11$ & 43 & $126-168$ \\
\hline 7140 & $2^{2} \cdot 3 \cdot 5 \cdot 7 \cdot 17$ & 16 & $140-153,167,168$ \\
\hline 7590 & $2 \cdot 3 \cdot 5 \cdot 11 \cdot 23$ & 12 & $167-178$ \\
\hline 7980 & $2^{2} \cdot 3 \cdot 5 \cdot 7 \cdot 19$ & 26 & $140-153,167-178$ \\
\hline 8190 & $2 \cdot 3^{2} \cdot 5 \cdot 7 \cdot 13$ & 38 & $130-153,167-180$ \\
\hline 8580 & $2^{2} \cdot 3 \cdot 5 \cdot 11 \cdot 13$ & 19 & $167-181,195,196,201,202$ \\
\hline 9240 & $2^{3} \cdot 3 \cdot 5 \cdot 7 \cdot 11$ & 14 & $168-181$ \\
\hline 9570 & $2 \cdot 3 \cdot 5 \cdot 11 \cdot 29$ & 8 & $174-181$ \\
\hline 9660 & $2^{2} \cdot 3 \cdot 5 \cdot 7 \cdot 23$ & 15 & $167-181$ \\
\hline 20910 & $2 \cdot 3 \cdot 5 \cdot 17 \cdot 41$ & 4 & $269-272$ \\
\hline 21930 & $2 \cdot 3 \cdot 5 \cdot 17 \cdot 43$ & 4 & $269-272$ \\
\hline 22230 & $2 \cdot 3^{2} \cdot 5 \cdot 13 \cdot 19$ & 4 & $269-272$ \\
\hline 22440 & $2^{3} \cdot 3 \cdot 5 \cdot 11 \cdot 17$ & 4 & $269-272$ \\
\hline
\end{tabular}


Table 2 (cont.)

\begin{tabular}{cccl}
\hline Champion & Decomposition & No. of appearances & The values of $Q$ \\
\hline 22610 & $2 \cdot 5 \cdot 7 \cdot 17 \cdot 19$ & 4 & $269-272$ \\
\hline 22770 & $2 \cdot 3^{2} \cdot 5 \cdot 11 \cdot 23$ & 4 & $269-272$ \\
\hline 23562 & $2 \cdot 3^{2} \cdot 7 \cdot 11 \cdot 17$ & 4 & $269-272$ \\
\hline 26334 & $2 \cdot 3^{2} \cdot 7 \cdot 11 \cdot 19$ & 4 & $269-272$ \\
\hline 27846 & $2 \cdot 3^{2} \cdot 7 \cdot 13 \cdot 17$ & 4 & $269-272$ \\
\hline 30030 & $2 \cdot 3 \cdot 5 \cdot 7 \cdot 11 \cdot 13$ & 87 & $231-233,269-352$ \\
\hline 31122 & $2 \cdot 3^{2} \cdot 7 \cdot 13 \cdot 19$ & 8 & $269-272,349-352$ \\
\hline 39270 & $2 \cdot 3 \cdot 5 \cdot 7 \cdot 11 \cdot 17$ & 56 & $269-272,349-400$ \\
\hline 40698 & $2 \cdot 3^{2} \cdot 7 \cdot 17 \cdot 19$ & 8 & $349-356$ \\
\hline 43890 & $2 \cdot 3 \cdot 5 \cdot 7 \cdot 11 \cdot 19$ & 17 & $349-356,399-407$ \\
\hline 53130 & $2 \cdot 3 \cdot 5 \cdot 7 \cdot 11 \cdot 23$ & 8 & $349-356$ \\
\hline
\end{tabular}

Table 3. The $H$-values for a given $Q$

\begin{tabular}{ccccccccc}
\hline$Q$ & $\left|D\left(\mathcal{M}_{Q}\right)\right|$ & $\frac{H_{1}(Q)}{H(Q)}$ & $\frac{H_{2}(Q)}{H(Q)}$ & $\frac{H_{3}(Q)}{H(Q)}$ & $\frac{H_{4}(Q)}{H(Q)}$ & $\frac{H_{5}(Q)}{H(Q)}$ & $\frac{H_{6}(Q)}{H(Q)}$ & $\frac{H_{7}(Q)}{H(Q)}$ \\
\hline 31 & 154 & 0.9733 & 0.0267 & 0 & 0 & 0 & 0 & 0 \\
\hline 32 & 162 & 0.9747 & 0.0253 & 0 & 0 & 0 & 0 & 0 \\
\hline 33 & 172 & 0.9636 & 0.0303 & 0.0061 & 0 & 0 & 0 & 0 \\
\hline 34 & 180 & 0.9408 & 0.0533 & 0.0059 & 0 & 0 & 0 & 0 \\
\hline 35 & 192 & 0.956 & 0.033 & 0.011 & 0 & 0 & 0 & 0 \\
\hline 100 & 1522 & 0.9059 & 0.0869 & 0.0065 & 0.0007 & 0 & 0 & 0 \\
\hline 101 & 1572 & 0.9161 & 0.079 & 0.0042 & 0.0007 & 0 & 0 & 0 \\
\hline 102 & 1588 & 0.9102 & 0.0829 & 0.0062 & 0.0007 & 0 & 0 & 0 \\
\hline 103 & 1639 & 0.9233 & 0.0707 & 0.0053 & 0.0007 & 0 & 0 & 0 \\
\hline 104 & 1663 & 0.9217 & 0.0718 & 0.0059 & 0.0007 & 0 & 0 & 0 \\
\hline 105 & 1687 & 0.9156 & 0.0759 & 0.0071 & 0.0006 & 0.0006 & 0 & 0 \\
\hline 106 & 1713 & 0.9108 & 0.0809 & 0.0071 & 0.0006 & 0.0006 & 0 & 0 \\
\hline 107 & 1766 & 0.9212 & 0.0714 & 0.0062 & 0.0012 & 0 & 0 & 0 \\
\hline 108 & 1784 & 0.9207 & 0.072 & 0.0061 & 0.0012 & 0 & 0 & 0 \\
\hline 109 & 1838 & 0.9278 & 0.0658 & 0.0059 & 0.0006 & 0 & 0 & 0 \\
\hline 110 & 1858 & 0.9216 & 0.0702 & 0.0076 & 0.0006 & 0 & 0 & 0 \\
\hline 311 & 14770 & 0.89 & 0.0954 & 0.0121 & 0.0023 & 0.0001 & 0.0001 & 0 \\
\hline 312 & 14818 & 0.8886 & 0.0965 & 0.0123 & 0.0025 & 0.0001 & 0.0001 & 0 \\
\hline 313 & 14974 & 0.8927 & 0.0934 & 0.0117 & 0.0021 & 0.0001 & 0.0001 & 0 \\
\hline 314 & 15052 & 0.8908 & 0.0954 & 0.0116 & 0.002 & 0.0001 & 0.0001 & 0 \\
\hline 315 & 15124 & 0.8898 & 0.0959 & 0.0119 & 0.0022 & 0.0001 & 0.0001 & 0 \\
\hline & & & & & & & &
\end{tabular}


Table 3 (cont.)

\begin{tabular}{ccccccccc}
\hline$Q$ & $\left|D\left(\mathcal{M}_{Q}\right)\right|$ & $\frac{H_{1}(Q)}{H(Q)}$ & $\frac{H_{2}(Q)}{H(Q)}$ & $\frac{H_{3}(Q)}{H(Q)}$ & $\frac{H_{4}(Q)}{H(Q)}$ & $\frac{H_{5}(Q)}{H(Q)}$ & $\frac{H_{6}(Q)}{H(Q)}$ & $\frac{H_{7}(Q)}{H(Q)}$ \\
\hline 316 & 15202 & 0.8892 & 0.0964 & 0.012 & 0.0022 & 0.0001 & 0.0001 & 0 \\
\hline 317 & 15360 & 0.8931 & 0.0934 & 0.0113 & 0.002 & 0.0001 & 0.0001 & 0 \\
\hline 318 & 15412 & 0.8914 & 0.0945 & 0.0118 & 0.0021 & 0.0001 & 0.0001 & 0 \\
\hline 319 & 15552 & 0.8906 & 0.0954 & 0.0117 & 0.0022 & 0.0001 & 0.0001 & 0 \\
\hline 320 & 15616 & 0.8903 & 0.0957 & 0.0117 & 0.0022 & 0.0001 & 0.0001 & 0 \\
\hline
\end{tabular}

\section{References}

[1] M. Balazard, Comportement statistique du nombre de facteurs premiers des entiers, in: Séminaire de Théorie des Nombres, Paris 1987-88, Progr. Math. 81, Birkhäuser, Boston, MA, 1990, 1-21.

[2] P. Erdős and E. G. Straus, Remarks on the differences between consecutive primes, Elem. Math. 35 (1980), 115-118.

[3] K. Ford, Distribution of integers with a divisor in an interval, in preparation.

[4] P. X. Gallagher, On the distribution of primes in short intervals, Mathematika 23 (1976), 4-9.

[5] -, Corrigendum (to [4]), ibid. 28 (1981), 86.

[6] R. R. Hall and G. Tenenbaum, On consecutive Farey arcs, Acta Arith. 44 (1984), 397-405.

[7] —, - Divisors, Cambridge Tracts in Math. 90, Cambridge Univ. Press, 1988.

[8] G. H. Hardy and S. Ramanujan, The normal number of prime factors of a number n, Quart. J. Math. 158 (1917), 76-92.

[9] R. Harley, Some estimates by Richard Brent applied to the "high jumpers" problem, unpublished manuscript, available at 〈http://pauillac.inria.fr/ harley/wnt.html $\rangle$.

[10] H.-K. Hwang, Sur la répartition des valeurs des fonctions arithmétiques. Le nombre de facteurs premiers d'un entier, J. Number Theory 69 (1998), 135-152.

[11] H. Nelson, Problem 654, J. Recreational Math. 11 (1978-79), 231.

[12] A. Odlyzko, M. Rubinstein and M. Wolf, Jumping champions, Experiment. Math. 8 (1999), 107-118.

[13] K. Rosen (ed.), Handbook of Discrete and Combinatorial Mathematics, CRC Press, 2000 .

[14] G. Tenenbaum, Sur la probabilité qu'un entier possède un diviseur dans un intervalle donné, Compositio Math. 51 (1984), 243-263.

[15] —, Un problème de probabilité conditionnelle en arithmétique, Acta Arith. 49 (1987), $165-187$.

Institute of Mathematics of the Romanian Academy

P.O. Box 1-764

Bucureşti 70700, Romania

E-mail: ccobeli@stoilow.imar.ro
Department of Mathematics University of Illinois at Urbana Champaign Urbana, IL 61801, U.S.A. E-mail: ford@math.uiuc.edu zaharesc@math.uiuc.edu

Received on 7.6.2002

and in revised form on 5.3.2003 